\title{
Microdialysis techniques in the study of brain and skeletal muscle
}

\author{
D.G. M aggs, W. P. B org, R .S. Sherwin \\ School of Medicine, Yale University, New Haven, USA
}

Summary Traditionally, plasma measurements have been used to monitor metabolic events and the actions of hormones that are actually taking place within tissue beds that are anatomically separated from the vascular compartment. It is generally assumed that the extracellular fluid (ECF) within metabolically active tissues is composed of substrates and hormones in concentrations that closely approximate those in plasma. Indeed, this view is implicit in nonsteady-state tracer calculations. However, through the use of microdialysis techniques in the study of tissue metabolism this view is being challenged. Our data suggest that there may be substantial concentration gradients for a variety of fuels between plasma and ECF, i.e. fuels (e.g. glucose) removed from the circulation being lower and fuels (e.g. glycerol, lactate, some amino acids) produced by tissues being higher than plasma levels. In short, the metabolic milieu seen by individual tissues (and hormone receptors?) may, at least in some instances, be strikingly different from that in plasma, and as a result, plasma measurements by themselves may not appropriately define the contributions of specific tissues to metabolic events, and overlook the importance of metabolic processes which are largely restricted to individual tissue beds. Through the use of microdialysis as a means of directly sampling ECF from metabolically important body tissues and with the evolution of its use in animal and human research, this technique will continue to offer exciting new insights into tissue metabolism and to investigate fundamental issues that cannot be addressed by other methods. [Diabetologia (1997) 40: S 75-S 82]

Keywords Brain, skeletal muscle, interstitial fluid.
Microdialysis offers a powerful investigative tool which enables in vivo sampling of extracellular fluid (ECF) from body tissues [1,2]. Alternative methods, such as positron emission tomography and magnetic resonance spectroscopy, enable insights into tissue metabolism, but are indirect and observations are derived. The Fick principle, through measurement of tissue blood flow and arteriovenous differences, is a more direct approach, however, it has anatomical limitations. In humans, this technique has mostly

Corresponding author: Dr. D. G. Maggs, Section of Endocrinology, Yale School of Medicine, P.O. Box 208020, New Haven, CT 06520-8020, USA

A bbreviations: ECF, Extracellular fluid; VMH, ventromedial hypothalamus; IDDM, insulin-dependent diabetes mellitus; 2DG, 2-deoxyglucose; GABA, $\gamma$-aminobutyric acid. been applied to the study of the limb as a means of 'isolating' skeletal muscle, but these studies are confounded by the contributions of other tissues to net metabolic activity [3]. Moreover, arteriovenous differences across a tissue bed assess 'net' changes but may underestimate the turnover of a substrate in the tissue in question. For example, in the limb, high rates of glycerol uptake and release occur, despite a small arteriovenous difference $[4,5]$.

To date, microdialysis has been used mainly in the study of animal brain, where the focus has been predominantly in the area of neurotransmission [6-8]. The application of microdialysis in humans has mostly been directed at fuel metabolism in adipose tissue although, more recently, it has also been applied to the study of skeletal muscle. From a local standpoint, we have applied microdialysis techniques to the in 
vivo study of both animal and human brain as well as the investigation of peripheral tissue metabolism in both skeletal muscle and adipose tissue. In animal brain, we have directed our attention to the role of the ventromedial hypothalamus (VMH) in glucose sensing and the effect of glucose availability on the release of neurotransmitters. Whereas, in the periphery, we have focused on human skeletal muscle, a tissue that plays a key role in fuel homeostasis. Through the unique access to specific tissue beds that microdialysis offers, we have applied this method in two main directions: (i) perturbing the local extracellular environment of a specific target tissue by the perfusion of substrates or pharmacologic agents via the microdialysis catheter, (ii) sampling local tissue ECF from the microdialysis catheter during systemic metabolic perturbations.

\section{M ethodologic considerations}

In view of our application of this technique in brain and peripheral tissues, it is important to address some general methodologic issues that concern the microdialysis process and some further, more specific, issues that we have encountered. Microdialysis is based on the principle of diffusion of substances across a permeable membrane between two compartments. Its typical use in vivo involves the perfusion of an isotonic artificial ECF solution (perfusate) into a tissue bed where perfusate equilibrates with the local tissue ECF across a dialysis membrane; this fluid (dialysate) is then collected for measurement of tissue substrates ex vivo. Although, through the nature of the microdialysis process, it also feasible to deliver substrates, pharmacologic agents or even hormones to the local tissue bed via the microdialysis catheter.

The performance or efficiency of a microdialysis system is dependent on a number of factors including the structural design of the microdialysis catheter. A schematic representation of the microdialysis system that we have used in peripheral tissues is shown in Figure 1, however, other similar prototypes exist, some with structural differences. Efficiency is also dependent on the nature of the dialysis membrane and a range of cellulose ester, polysulfate and polycarbonate membranes are in use, each with specific physical characteristics. It is theorized that the nature of the perfusate may also influence the dialysis process and that to minimize artifact caused by the perfusate itself it is recommended that catheters should be perfused with a solution that closely resembles tissue ECF. For this reason, we use an artificial ECF solution $(135 \mathrm{mmol} / 1$ $\mathrm{NaCl}, 3 \mathrm{mmol} / \mathrm{l} \mathrm{KCl}, 1 \mathrm{mmol} / \mathrm{l} \quad \mathrm{MgCl}_{2}, \mathrm{CaCl}_{2}$ $1.2 \mathrm{mmol} / \mathrm{l}$ and sodium phosphate to buffer the perfusate to $\mathrm{pH}$ 7.4) which closely approximates tissue ECF.

In vivo, the concentration of substances in dialysate is invariably a fraction of the actual ECF

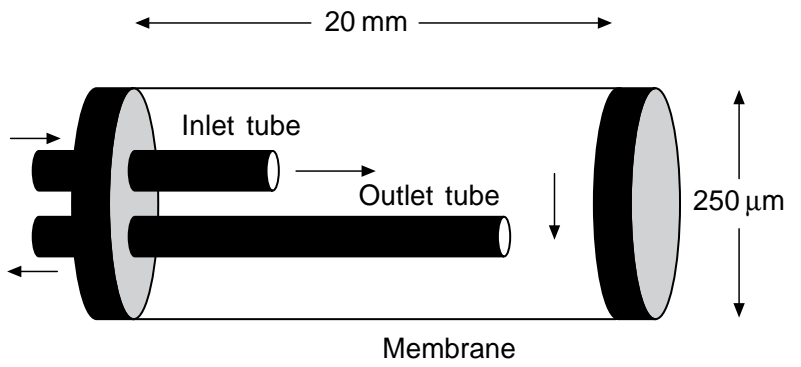

Fig. 1. Schematic representation of the microdialysis probe we utilize in the study of adipose and skeletal muscle tissues. Fluid is perfused (perfusate) via the inlet tube into the cylindrical chamber comprizing the dialysis membrane and subsequently exits via the outlet tube (dialysate)

concentration and this reflects the 'recovery' of the microdialysis system. Recovery is often expressed as a percent (i.e. [dialysate]/[ECF] $\times 100$ ) and is easily calculated in vitro by placing a microdialysis catheter in an aqueous solution containing a known amount of substrate. The catheter is perfused at a constant rate and substrate concentration is measured in dialysate enabling the calculation of in vitro recovery (i.e. [dialysate]/[solute] $\times 100)$. In vivo recovery is generally less than that seen in vitro, and this is thought to reflect the reduced capacity for substrates and other molecules to diffuse in the ECF space surrounding the membrane when compared with diffusing capacity in an aqueous solution in vitro. However, it is possible to calculate absolute ECF concentrations, and therefore in vivo recovery, by a number of methods, two of which we have utilized in our studies: the "no net flux' [1] and 'zero flow rate' [9] procedures. Both of these methods have been validated in the past and we have utilized both procedures in vitro and in vivo. The no net flux method is based on the principle of perfusing a microdialysis system with different concentrations and, by measuring dialysate concentrations of substrate, it is possible to calculate a steady-state where perfusate concentration equals ECF concentration, in other words, a steady-state of "no net flux'. This estimate represents absolute ECF concentrations of the substrate in question. Figure 2 shows representative examples of 'no net flux' calibration procedures carried out in healthy human subjects for the measurement of glucose in the ECF space of adipose tissue and skeletal muscle.

The 'zero flow rate' method is based on the principle that the relative recovery of substrate across dialysis membrane is inversely related to perfusate flow through the microdialysis system, and when flow rate is zero the perfusate and ECF are in a state of complete equilibrium. Therefore, by measuring dialysate concentrations of substrate at different perfusate flow rates, it is possible to plot the inverse relationship and to extrapolate to zero flow rate by non-linear regression, thus estimating absolute concentration of 


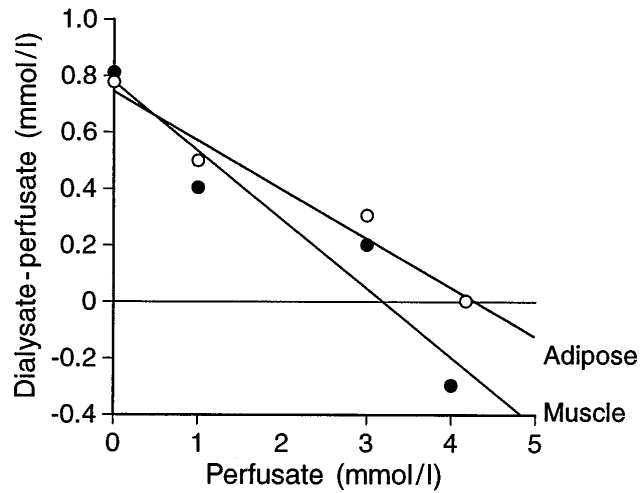

Fig. 2. Data from 'no net flux' calibration procedures carried out in a non-obese human subject. Data indicates glucose concentrations ( $\mathrm{mmol} / \mathrm{l})$ expressed as the difference between dialysate and perfusate concentrations on the $y$-axis, plotted against perfusate concentration on the $\mathrm{X}$-axis. Data is plotted from adipose $(\bigcirc)$ and skeletal muscle $(O)$ dialysate at four different perfusate concentrations of glucose ( $\mathrm{mmol} / \mathrm{l})$. As indicated, a regression line is extrapolated through the four data points for each tissue bed and the estimate of absolute ECF concentration is the point where the line crosses the point of [dialysate]-[perfusate] $=0$ on the $y$-axis, the point of no net flux
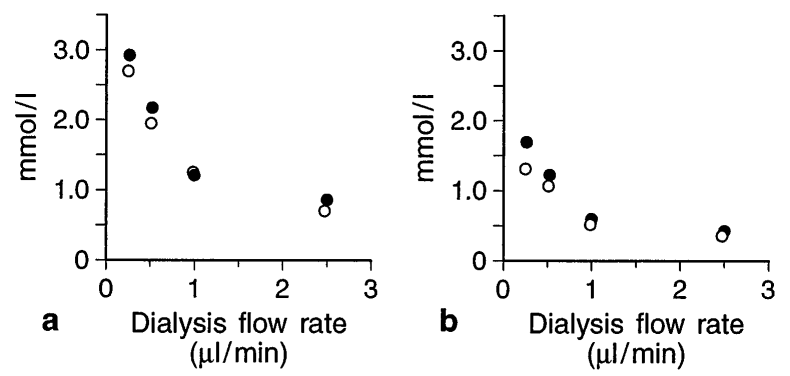

Fig. 3. Data from a 'zero flow rate' calibration procedure carried out in a non-obese human subject. Data indicates (a) glucose and (b) lactate concentrations ( $\mathrm{mmol} / \mathrm{l})$ in adipose $(\bigcirc)$ and skeletal muscle $(O)$ dialysate at different dialysis flow rates $(\mu 1 / \mathrm{min})$

substrate in ECF. Figure 3 shows representative examples of 'zero flow rate' calibration procedures carried out in healthy humans to measure glucose and lactate concentrations in adipose tissue and skeletal muscle ECF. In addition, as further validation of these techniques, we have shown that estimates of peripheral tissue ECF concentrations of glucose, lactate and some key amino acids by these two methods are virtually identical.

A significant drawback with these calibration techniques is that they are inherently time consuming and the more recent description of an alternate calibration technique $[10,11]$ involving isotopic perfusion is of interest as this method allows an almost 'immediate' estimate of microdialysis recovery. The technique is based on the principle that through the addition of substrate in isotope form to the perfusate, the relative delivery of isotopic substrate from perfusate into local tissue ECF equals the relative recovery of cold substrate from tissue ECF into dialysate. This method seems to compare favourably with the no net flux calibration procedure; however, data produced by this technique raise some methodologic concerns [11]. Apparent overestimates of ECF glucose levels in rat adipose tissue measured by this technique can be explained by an accumulation of isotope in the local tissue surrounding the microdialysis catheter thereby resulting in an underestimate of the delivery of glucose isotope to the tissue and artificially overestimating calculations for cold levels of glucose in ECF. Finally, with the question of measuring absolute ECF levels of susbstrates in mind, Arner and colleagues are utilizing a microdialysis system which they propose has an approximate $100 \%$ relative recovery [12]. In other words, dialysate concentrations of substrate are equal to those in ECF. This high efficiency is allowed because the microdialysis catheters have a large surface area of dialysis membrane (30 mm length) and are perfused at very low rates $(0.3 \mu \mathrm{l} / \mathrm{min})$.

A further concern with microdialysis studies conducted over many hours is the 'stability' of the microdialysis system. In other words, could changes in substrate concentrations observed during study interventions be a manifestation of an artifact created by the probe itself resulting in a shifting baseline? This is an area of some contention as it has been suggested by some that microdialysis may cause a depletion of substrate from the local tissue bed causing a time-dependent shift in dialysate substrate concentration [1]. However, others contest that this phenomenon is not a significant concern [13]. Furthermore, this potential phenomenon has only been described for glucose and not for other substrates. To attempt to address this issue, we carried out a series of studies in healthy subjects under basal conditions and found no significant time-dependent change in skeletal muscle and adipose tissue dialysate concentrations of substrates under basal conditions over a $6 \mathrm{~h}$ period.

An additional concern with the microdialysis process is the possible artifact that locally altered blood flow may have on the recovery of ECF substrates. A recent study, involving the coupling of laser Doppler flowmetry as a measure of local blood flow with microdialysis, showed that insertion of the microdialysis catheter into skin caused a short lived hyperaemic response which quickly ( $<15 \mathrm{~min}$ ) resolved [14]. Thus, in the bounds of the short-term metabolic studies we and others conduct, the catheter itself does not seem to significantly alter local blood flow. However, in the context of metabolic studies involving hyperinsulinaemia, especially with concurrent hypoglycaemia, where one would expect physiologic alterations in blood flow it is important to consider the potential influence that blood flow may have on the recovery of 
substrates by microdialysis. This issue was first raised when ethanol clearance from microdialysis perfusate was developed and validated [15] as a method of attaining a qualitative measure of local tissue blood flow at the site of microdialysis catheter insertion. Studies demonstrated that, in animals, gross changes in tissue blood flow alter the recovery of glucose by microdialysis [16] (i.e. an increase in local blood flow increases dialysate recovery of glucose) and similar findings have since been reported in human adipose and muscle tissues but with a less predictable effect of altered blood flow on the recovery of ECF lactate [17].

To address this issue, we studied the potential effects that insulin-induced hypoglycaemia may have on the recovery of ECF glucose [18]. We studied healthy subjects during hypoglycaemic clamps (plasma glucose $2.8 \mathrm{mmol} / \mathrm{l}$ ) and measured ECF glucose levels in peripheral tissues by two different methods. On one occasion, we indirectly estimated tissue ECF glucose during hypoglycaemia by calculating absolute levels of ECF glucose at baseline and therefore the in vivo recovery of our microdialysis system. We then applied the baseline recovery to indirectly calculate ECF glucose during a hypoglycaemic clamp ([hypoglycaemia dialysate glucose] $\times$ baseline recovery $=$ [hypoglycaemic ECF glucose $]$ ). On the second occasion, we made direct estimations of absolute ECF glucose concentrations during stable hypoglycaemia using the no net flux calibration procedure. If the microdialysis recovery of glucose had been altered by hypoglycaemia itself, then the indirect and direct estimates of tissue ECF glucose during hypoglycaemia would differ. However, we found no difference between the indirect and direct estimations of ECF glucose levels under these conditions, therefore suggesting that the relative recovery of glucose from the tissue bed by microdialysis was not altered in this particular model of insulin-induced hypoglycaemia. In contrast to these findings, the blood flow and ECF glucose changes that occur during insulin-induced hypoglycaemia have been described in some detail in rat muscle and adipose tissue with evidence that hypoglycaemia-induced blood flow changes may affect microdialysis recovery of glucose [19], however, these studies were done under non-steady-state conditions.

\section{Brain}

Although the vast majority of work in the field of microdialysis has been in animal brain, little attention has been directed to the study of brain fuel homeostasis and, more specific to our area of interest, the role of the brain as a glucose sensor under conditions of hypoglycaemia. The recent report of the DCCT Research Group [20] demonstrated the long-term benefits of intensified treatment in insulin-dependent diabetes mellitus (IDDM) and, as a result, this therapy has been recommended for, and undoubtedly will be offered to, an increasing number of IDDM patients in an effort to prevent or delay microvascular and neuropathic complications. Unfortunately, the frequency and severity of hypoglycaemia is markedly increased by such regimens [21]. In light of this, the neurohormonal response to hypoglycaemia in health and diabetes has been extensively investigated in recent years, resulting in a better understanding of the defective counterregulatory mechanisms that exist in IDDM. However, it is still not clear what mechanisms are involved in glucose sensing and the initiation of the neurohormonal response to hypoglycaemia.

A role for both cerebral and extra-cerebral glucose sensors has been proposed [22,23]. Although there is some evidence that the liver may play a role in the activation of sympathoadrenal activity [24], most data point to the central nervous system as the dominant centre for the sensing and integration of hypoglycaemic signals. This view is perhaps best supported by studies showing that bilateral infusion of glucose into the carotid and vertebral arteries (maintaining intracerebral euglycaemia in the face of systemic hypoglycaemia) nearly abolishes hormone release during hypoglycaemia $[22,25]$. These studies, however, do not define the precise brain regions involved. While the hypothalamus has long been viewed as the most likely site [26], others have suggested that the glucose sensor is located in the hindbrain or spinal cord [27, 28]. Recent data from our laboratory provide strong evidence that the VMH is essential for hypoglycaemic counterregulation [29]. We observed that conscious VMH-lesioned rats (bilateral lesions produced by local ibotenic acid injection 2 weeks earlier) had blunted glucagon, epinephrine and norepinephrine responses to a hypoglycaemic stimulus $(3 \mathrm{mmol} / \mathrm{l}$ or $2.5 \mathrm{mmol} / \mathrm{l})$ when compared with responses noted in controls. We therefore reasoned that, if glucosensors are in the VMH, glucopenia limited to this centre should cause counterregulatory responses despite peripheral normoglycaemia. At this point, we applied microdialysis techniques to study the role of the $\mathrm{VMH}$ in triggering counterregulatory responses to hypoglycaemia [30]. In the $\mathrm{VMH}$ or frontal lobes (controls) of awake rats $(n=20)$, 2-deoxyglucose (2-DG) or glucose (to serve as a control) was perfused via microdialysis catheters to produce localized cellular glucopenia. Perfusion of 2-DG (but not glucose) into the VMH caused a striking increase in plasma glucagon (by $200 \%$ ), epinephrine (by $600 \%$ ), norepinephrine (by $400 \%$ ) in association with a prompt elevation of circulating blood glucose. It should also be noted that 2-DG had no effect when delivered to the frontal lobes. These data therefore suggest that the neurons sensing glucopenia are in the VMH. To follow the findings from this study, we have used an opposite experimental paradigm 
again to determine the role of VMH in glucose sensing. Under conditions of systemic hypoglycaemia, we used microdialysis to perfuse the VMH bilaterally with glucose preventing VMH hypoglycaemia [31]. Data indicate that hypoglycaemic rats with 'euglycaemic' VMH have markedly diminished hormonal responses to systemic hypoglycaemia, again suggesting that the VMH is the dominant glucose sensor for activation of the counterregulatory response to hypoglycaemia.

A concern with these experiments relates to whether the anatomical positioning of the catheters was appropriate and whether the local perfusion with 2DG confined its effect to the VMH. The hypothalamus is a small, albeit highly complex, area of the brain and, despite positioning the catheters with stereotactic surgery, one must be careful in attributing these findings to an area as small as the VMH. However, the appropriate anatomical localization of the catheters was confirmed histologically. In addition, in a small number of rats $(n=4)$, further studies were catheters performed where $\left[{ }^{3} \mathrm{H}\right] 2-\mathrm{DG}$ was perfused via the catheters and local radioactivity was measured in $\mathrm{VMH}$ and surrounding structures. This procedure confirmed that radioactivity was largely restricted to the $\mathrm{VMH}$; and the radioactivity in the surrounding tissues was only slightly above background levels. In other words, the local perfusion of glucose via the microdialysis catheters was indeed confined to the VMH.

The cellular mechanisms used by the $\mathrm{VMH}$ to sense and transduce the glucose signal are unknown. It is intriguing to speculate that the $\mathrm{VMH}$ glucose sensor shares common features with the only glucose sensing cell that has been well characterized, the pancreatic beta cell. Recent studies support this view. Jetton et al. [32] have reported that cells within the medial hypothalamus express glucokinase as well as GLUT2. Furthermore, VMH neurons contain ATPsensitive potassium channels, a key signal transduction protein in beta cells [33]. In keeping with this hypothesis, we have recently reported, again through the utilization of microdialysis catheters in rat substantia nigra, a brain region rich in $\mathrm{K}_{\mathrm{ATP}}$ channels as determined by sulfonylurea binding [33], that this area responds to changes in local glucose availability via effects on $\mathrm{K}_{\mathrm{ATP}}$ channels. Perfusing the dialysis catheter with $10 \mathrm{mmol} / \mathrm{l}$ glucose increased $\gamma$-aminobutyric acid (GABA) release two-fold. Local perfusion with the sulfonylurea glipizide had a nearly identical effect; whereas perfusion with the specific $\mathrm{K}_{\mathrm{ATP}}$ channel activator, lemakalim, or 2-DG with oligomycin inhibited GABA release by $45-50 \%$. The latter effect was blocked by glipizide. When systemic hypoglycaemia was produced in awake rats, nigral dialysate GABA concentrations decreased by approximately $50 \%$, as well. These data suggest that glucose may modulate nigral GABA release via an effect on $\mathrm{K}_{\mathrm{ATP}}$ channels, a pattern which bears a striking similarity to that of pancreatic beta cells. Thus, glucose could act as a signalling molecule for at least some neurons in the central nervous system. Taken together, these observations suggest that the $\mathrm{VMH}$ might function much like the beta cell, a scenario that might account for functional changes in hormone release seen with chronic hyper- or recurrent hypoglycaemia.

It is well established that glucose passes across the blood brain barrier by carrier mediated transport (via GLUT 1 transporters) and unsaturable (diffusion) mechanisms [34]. Once in the ECF, the concentration of glucose seen by neurones and glia is, however, probably much lower than that of plasma. Estimates of brain ECF glucose levels in animals using microdialysis or microelectrodes range from $0.5-$ $2.8 \mathrm{mmol} / \mathrm{l}[35,36]$, suggesting that a steep glucose concentration gradient exists between blood and brain ECF. We have recently had the unique opportunity to utilize microdialysis to measure glucose levels in brain ECF of conscious humans undergoing intracerebral depth electrode monitoring for intractable epilepsy [37]. Preliminary data suggest that ECF glucose levels in human brain are very different from plasma levels; i.e. approximately $30 \%$ of plasma levels. These findings imply that neurons are bathed in a metabolic milieu different to that seen in the circulation.

\section{Skeletal muscle}

Application of microdialysis techniques to the study of peripheral tissues has increased in recent years and this technique is particularly well suited to these tissues since larger catheters can be used (than those used in the study of animal brain), substantially increasing the efficiency of the microdialysis process and therefore the ability to detect metabolic change in ECF. Most attention has been directed to adipose tissue, mainly because of easy access, and studies have reported the measurement of ECF levels of glucose, lactate and glycerol $[1,38-42]$ and characterized the opposing effects of insulin and sympathetic stimulation on adipose tissue fat and glucose metabolism $[39,43-45]$. The application of microdialysis to the study of diabetic or insulin resistant states has been limited but work in this area includes study of the effects of insulin withdrawal [45] and hyperglycaemia [46] on levels of substrates in adipose ECF in IDDM; and abnormalities of glucose and glycerol metabolism in adipose tissue have also been reported in obesity [39, 47], patients with liver cirrhosis [48] and spinal cord injured patients with mild insulin resistance [49].

Microdialysis has been applied to a lesser extent in skeletal muscle; a tissue that plays a major role in protein metabolism and insulin-mediated peripheral 
glucose uptake, and that also serves as the major source of gluconeogenic precursors under catabolic conditions. In rats, a number of studies have shown that microdialysis is applicable to this tissue bed [15, $19,50]$ and we have used microdialysis in both skeletal muscle and adipose tissue ECF in humans. Our studies were firstly notable for the concentration gradients that exist for key metabolic substrates between the ECF compartment of both tissue beds and the circulation. High glycerol levels were noted in skeletal muscle dialysate, suggesting that there is significant lipolysis occurring in skeletal muscle and that the importance of the glucose-fatty acid cycle may be underestimated in this tissue bed [51]. In addition, we also demonstrated that ECF levels of lactate were higher than plasma levels indicating that there is an efflux of lactate from both tissues under fasting conditions. Whereas, levels of glucose in muscle and adipose ECF were approximately $30 \%$ lower than arterialized plasma levels indicating a concentration gradient in the opposite direction in keeping with glucose uptake.

Peripheral tissue ECF glycerol levels were higher than levels reported by others [41, 42] raising the possibility that the high glycerol levels were, in part, artifactual. However, in vitro, we confirmed that the glycerol content of the cuprophan membrane we used was seemingly negligible. This fact was further underlined when we used the same catheters in human brain and found that glycerol levels were immeasurably low in brain dialysate, a tissue bed where you would expect negligible lipolytic activity. In addition, the question that elevated glycerol levels in dialysate may be a trauma artifact has been largely refuted by past work showing that the initial trauma reaction quickly subsides shortly after catheter implantation [13] and, thereafter, dialysate sampling reflects a stable ECF environment for hours. Finally, specific to skeletal muscle, the disparity between muscle ECF and plasma glycerol raises the question of whether the muscle probes were positioned appropriately as the presence of high ECF glycerol could imply that the muscle catheter was in subcutaneous fat rather than muscle itself. However, we studied non-obese subjects and MRI scans of the sites of catheter insertion confirmed that the muscle catheters were positioned correctly. Moreover, we demonstrated that taurine, alanine and glutamine levels were higher in muscle than in adipose tissue dialysate, supporting the view that the muscle and adipose catheters were indeed in two distinct tissues. Contrary to our observations, Samra et al. [52] stated that skeletal muscle tissue ECF levels of glycerol must be near to plasma levels on the basis of observing a net uptake of glycerol across the limb following a square-wave intravenous infusion of glycerol, but without directly measuring ECF levels by microdialysis. On the other hand, using a microdialysis system with approximate
$100 \%$ recovery, Enoksson et al. [12] reported that basal ECF levels of glycerol are indeed higher than plasma in skeletal muscle as well as adipose tissue but absolute levels were not nearly as high as those observed by us. We have since tested our original microdialysis system side by side with a commercially available system in fasted humans during a continuous infusion of stable isotope of glycerol to attain a steadystate isotope enrichment of glycerol in both plasma and adipose tissue ECF. Our preliminary data show that with both microdialysis systems the isotope enrichment in the adipose dialysate was approximately $10-20 \%$ of plasma enrichments indicating local glycerol production. However, the relative enrichment of isotope in dialysate was at least twofold higher (and therefore nearer to plasma enrichments) in the commercially available catheter when compared with our original catheter. Accepting these observations are confined to adipose tissue and that the commercially available microdialysis catheter generated data that supports observations made by others, this would suggest our original microdialysis system may have overestimated glycerol levels in skeletal muscle and adipose tissues.

A further interesting development in the use of microdialysis in the study of peripheral tissues is the application of stable or radioactive isotopes in the study of local tissue metabolism. As mentioned earlier, isotopes added to the perfusate have been used as a measure of microdialysis recovery $[10,11]$ but a more recent development has been the addition of substrate isotope to perfusate and the measurement of the substrate's metabolite in its isotope form in dialysate. Henry et al. [53] recently reported the perfusion of $\mathrm{U}^{13} \mathrm{C}$ glucose via a microdialysis catheter into human adipose tissue and the measurement of ${ }^{13} \mathrm{C}$ lactate in tissue dialysate as an indicator of local anaerobic glycolysis [53]. This particular application of microdialysis techniques may offer a unique insight into local tissue metabolic processes.

\section{References}

1. Lonnroth P, Jansson P-A, Smith U (1987) A microdialysis method allowing characterisation of intercellular water space in humans. Am J Phys 253: E228-E231

2. Ungerstedt U (1991) Microdialysis - principles and applications for studies in animals and man. J Int Med 230: 365-373

3. Simonsen L, Bulow J, Madsen J et al. (1993) Local forearm and whole body respiratory quotient in humans after an oral glucose load. Acta Physiol Scand 147: 69-75

4. Coppack S, Judd R, Miles J (1993) Uptake and release of glycerol and free fatty acids by human adipose and muscle tissue in vivo. Diabetes 42 [Suppl 1]: 38A (Abstract)

5. Elia M, Kahn K, Calder G et al. (1993) Glycerol exchange across the human forearm assessed by a combination of tracer and arteriovenous exchange techniques. Clin Sci 84: 99104 
6. Hamberger A, Jacobson I, Nystrom B et al. (1991) Microdialysis sampling of the neuronal environment in basic and clinical research. J Int Med 230: 375-380

7. Morrison P, Bungay P, Hsiao J et al. (1991) Quantitative microdialysis. In: Robinson TE, Justice JB Jr (eds) Microdialysis in the neurosciences. Elsevier, New York, pp 47-80

8. During M, Craig J, Hernandez T et al. (1992) Effect of amygdala kindling on the in vivo release of GABA and 5$\mathrm{HT}$ in the dorsal raphe nucleus in freely moving rats. Brain Res 584: 36-44

9. Jacobson I, Sandberg M, Hamberger A (1985) Mass transfer in brain dialysis devices - a new method for the estimation of extracellular amino acids concentration. J Neurosci Meth 15: 263-268

10. Jansson P, Veneman T, Nurjhan N et al. (1994) An improved method to calculate adipose tissue interstitial substrate recovery for microdialysis studies. Life Sci 54: 16211624

11. Lonnroth P, Strindberg L (1995) Validation of the 'internal reference technique' for calibrating microdialysis catheters. Acta Physiol Scand 153: 375-380

12. Enoksson E, Hagstrom-Toft E, Arner P (1996) Role of phosphodiesterase-III in the antilipolytic effect of insulin in human adipose versus skeletal muscle. Int J Obesity 20 [Suppl 4]: 84 (Abstract)

13. Bolinder J, Hagstrom E, Ungerstedt U et al. (1989) Microdialysis of subcutaneous adipose tissue in vivo for continuous glucose monitoring in man. Scand J Clin Lab Invest 49: $465-474$

14. Anderson C, Andersson T, Wardell K (1994) Changes in skin circulation after insertion of a microdialysis probe visualized by laser Doppler flowmetry. J Invest Dermatol 102: 807-811

15. Hickner R, Bone D, Ungerstedt U et al. (1994) Muscle blood flow during intermittent exercise: comparison of the microdialysis ethanol technique and ${ }^{133} \mathrm{Xe}$ clearance. Clin Sci 86: $15-25$

16. Hickner R, Rosdahl H, Borg I et al. (1991) Ethanol may be used with the microdialysis technique to monitor blood flow changes in skeletal muscle: dialysate glucose concentration is blood-flow-dependent. Acta Physiol Scand 143: 355-356

17. Rosdahl H, Ungerstedt U, Jorfeldt L et al. (1993) Interstital glucose and lactate balance in human skeletal muscle and adipose tissue studied by microdialysis. J Physiol - London 471: 637-657

18. Maggs D, Jacob R, Rife F et al. (1997) Counterregulation in peripheral tissues: effect of systemic hypoglycaemia on levels of substrates and catecholamines in human skeletal muscle and adipose tissue. Diabetes 46: 70-76

19. Fuchi T, Rosdahl H, Hickner R et al. (1994) Microdialysis of rat skeletal muscle and adipose tissue: dynamics of the interstitial glucose pool. Acta Physiol Scand 151: 249-260

20. The Diabetes Control and Complications Trial Research Group (1993) The effect of intensive treatment of diabetes on the development and progression of long term complications in insulin-dependent diabetes mellitus. $\mathrm{N}$ Eng $\mathrm{J}$ Med 32: 977-986

21. The Diabetes Control and Complications Trial Research Group (1991) Epidemiology of severe hypoglycaemia in the diabetes control and complications trial. Am J Med 90: 450-459

22. Biggers D, Myers S, Neal D et al. (1989) Role of the brain in counterregulation of insulin-induced hypoglycaemia in dogs. Diabetes 37: 7-16

23. Lautt W (1980) Hepatic nerves: a review of their functions and effects. Can J Physiol Pharmacol 58: 105-123
24. Donovan C, Halter J, Bergman R (1991) Importance of hepatic glucoreceptors in sympathoadrenal response to hypoglycaemia. Diabetes 40: 155-158

25. Frizzel R, Jones E, Davis S et al. (1993) Counterregulation during hypoglycaemia is directed by widespread brain regions. Diabetes 42: 1253-1261

26. Frohman L, Nagai K (1976) Central nervous system-mediated stimulation of glucagon secretion in the dog following 2-deoxy-glucose. Metabolism 25: 1449-1452

27. DiRocco R, Grill H (1979) The forebrain is not essential for sympathoadrenal hyperglycemic response to glucoprivation. Science 204: 1112-1114

28. Ritter R, Slusser P, Stone S (1981) Glucoreceptors controlling feeding and blood glucose: location in the hindbrain. Science 213: 451-453

29. Borg W, During M, Sherwin R et al. (1994) Ventromedial hypothalamic lesions in rats suppress counterregulatory responses to hypoglycaemia. J Clin Invest 93: 1677-1682

30. Borg W, Sherwin R, During M et al. (1995) Local ventromedial hypothalamus glucopenia triggers counteregulatory hormone release. Diabetes 44: 180-184

31. Borg M, Sherwin R, Borg W et al. (1997) Local ventromedial hypothalamus glucose perfusion blocks counterregulation during systemic hypoglycaemia in awake rats. J Clin Invest 99: 361-365

32. Jetton T, Liang Y, Pettepher C et al. (1994) Analysis of upstream glucokinase promoter activity in transgenic mice and identification of glucokinase in rare neuroendocrine cells in the brain and gut. J Biol Chem 269: 3641-3654

33. Zini S, Tremblay E, Roison M et al. (1991) Regional distribution of sulfonylurea receptors in the brain of rodent and primate. Brain Res 542: 151-154

34. Siesjo B (1988) Hypoglycaemia, brain metabolism and brain damage. Diab Met Rev 4: 113-144

35. Lund-Andersen H (1979) Transport of glucose from blood to brain. Physiol Rev 59: 305-352

36. Fellows L, Boutelle M, Fillenz M (1992) Extracellular brain glucose levels reflect local neuronal activity: a microdialysis study in awake, freely moving rats. J Neurochem 59: 2141-2147

37. Wallace E, During M, Sherwin R (1994) Direct measurement of interstitial glucose concentration in the human brain: effect of changing circulating glucose. Diabetes 43 [Suppl 1]: 47A (Abstract)

38. Jansson P, Fowelin J, Smith U et al. (1988) Characterisation by microdialysis of intercellular glucose level in subcutaneous tissue in humans. Am J Physiol 255: E218-E220

39. Hagstrom E, Arner P, Ungerstedt U et al. (1990) Subcutaneous adipose tissue: a source of lactate production after glucose ingestion in humans. Am J Physiol 258:E888E893

40. Jansson P, Smith U, Lonnroth P (1990) Evidence for lactate production by human adipose tisue in vivo. Diabetologia 33: $253-256$

41. Jansson P, Smith U, Lonnroth P (1990) Interstitial glycerol concentration measured by microdialysis in two subcutaneous regions in humans. Am J Phys 258: E918-E922

42. Arner P, Kriegholm E, Engfeldt P (1990) In situ studies of catecholamine-induced lipolysis in human adipose tissue using microdialysis. J Pharm Exp Ther 254: 284-288

43. Arner P, Kriegholm E, Engfeldt P et al. (1990) Adrenergic regulation of lipolysis in situ at rest and during exercise. $\mathrm{J}$ Clin Invest 85: 893-898

44. Arner P, Kriegholm E, Engfeldt P (1991) In vivo interactions between beta- 1 and beta- 2 adrenoceptors regulate catecholamine tachyphylaxia in human adipose tissue. $\mathrm{J}$ Pharm Exp Ther 259: 317-322 
45. Hagstrom-Toft E, Arner P, Johansson U et al. (1992) Effect of insulin on human adipose tissue metabolism in situ. Interactions with beta-adrenoceptors. Diabetologia 35: 664670

46. van der Merwe T, Eklund L, Jansson P et al. (1994) Effects of hyperglycemia on in vivo adipose tissue metabolism studied with microdialysis in IDDM subjects. Diabetes 43: 866-780

47. Jansson P, Larsson A, Smith U et al. (1992) Glycerol production in subcutaneous adipose tissue in lean and obese humans. J Clin Invest 89: 1610-1617

48. Johansson U, Arner P, Bolinder J et al. (1993) Influence of insulin on glucose metabolism and lipolysis in adipose tissue in situ in patients with liver cirrhosis. Eur J Clin Invest 23: 837-844

49. Karlsson A, Attvall S, Jansson P et al. (1995) Influence of the sympathetic nervous system on insulin sensitivity and adipose tissue metabolism: a study in spinal cord-injured subjects. Metabolism 44: 55-58
50. Yang W, Oshida Y, Wu W et al. (1995) Effect of daily voluntary running on in vivo insulin action in rat skeletal muscle and adipose tissue as determined by microdialysis technique. Int J Sports. Med. 16: 99-104

51. Maggs D, Jacob R, Rife F et al. (1995) Interstitial concentrations of glycerol, glucose and amino acids in human quadricep muscle and adipose tissue. J Clin Invest 96: 370-377

52. Samra J, Ravell C, Giles SL et al. (1996) Interstitial glycerol concentration in human skeletal muscle and adipose tissue is close to the concentration in blood. Clin Sci 90: 453-456

53. Henry S, Schneiter P, Jequier E et al. (1995) Effects of hyperglycemia and hyperinsulinaemia on lactate production by subcutaneous abdominal adipocytes. Diabetologia 38 [Suppl 1]: A12 (Abstract) 